\title{
A Low Cost Shading Analyzer and Site Evaluator Design to Determine Solar Power System Installation Area
}

\author{
Selami Kesler, ${ }^{1}$ Sinan Kivrak, ${ }^{2}$ Hilmi Gurleyen, ${ }^{3}$ Furkan Dincer, ${ }^{4}$ \\ Saban Yilmaz, ${ }^{5}$ and Hasan Riza Ozcalik ${ }^{5}$ \\ ${ }^{1}$ Department of Electrical and Electronics Engineering, Pamukkale University, 20070 Denizli, Turkey \\ ${ }^{2}$ Department of Energy Systems Engineering, Yildirim Beyazit University, 06830 Ankara, Turkey \\ ${ }^{3}$ Department of Electrical Engineering, Yildiz Technical University, 34349 Istanbul, Turkey \\ ${ }^{4}$ Department of Computer Engineering, Mustafa Kemal University, 36200 Hatay, Turkey \\ ${ }^{5}$ Department of Electrical and Electronics Engineering, Kahramanmaras Sutcu Imam University, 46100 Kahramanmaras, Turkey
}

Correspondence should be addressed to Selami Kesler; skesler@pau.edu.tr

Received 9 October 2014; Revised 17 December 2014; Accepted 17 December 2014

Academic Editor: Elias Stathatos

Copyright (C) 2015 Selami Kesler et al. This is an open access article distributed under the Creative Commons Attribution License, which permits unrestricted use, distribution, and reproduction in any medium, provided the original work is properly cited.

Shading analyzer systems are necessary for selecting the most suitable installation site to sustain enough solar power. Afterwards, changes in solar data throughout the year must be evaluated along with the identification of obstructions surrounding the installation site in order to analyze shading effects on productivity of the solar power system. In this study, the shading analysis tools are introduced briefly, and a new and different device is developed and explained to analyze shading effect of the environmental obstruction on the site on which the solar power system will be established. Thus, exposure duration of the PV panels to the sunlight can be measured effectively. The device is explained with an application on the installation area selected as a pilot site, Denizli, in Turkey.

\section{Introduction}

The importance of carbon-free energy generation was recognized; solar electricity or photovoltaic (PV) technology has attracted attention as a potentially widespread approach to sustainable energy generation [1-3]. However, the evaluation of power losses in solar cells is a key issue in identifying technological and material problems. By the way, PV panels and features of the installation site greatly affect the performance of solar power system [2]. On the other hand, efficiency of the solar power system can be increased through many measurements and investigations. One of the different studies on efficient improvement of PV power system is to use a new energy storage technology used in the plant due to wide range of power outputs, high storage efficiency, rapid response, low maintenance cost, and long lifecycle [4]. Some of the most important investigations in terms of improving the power system are the evaluation of the installation site, analysis of the shading resulting from the environmental obstructions, and weather conditions [5].
Temporary shading elements such as leaves in forested areas, snow in mountainous areas, bird droppings, and air pollution are not significant factors as long as the system is self-clean. In addition, some of the array arrangements established vertically or horizontally may reduce the effects of air conditions. More often than that, the shading caused by snow affects only a couple of PV panel rows. However, distant or close mountains, fast growing trees, and tall buildings are considerable factors compared to temporary shaders. Settlements and architectures of the buildings have become more important in large cities during the progress of urbanization, particularly at the point of effective usage of the sun. The distance between large buildings and their heights restrict the available direct sunlight. There are some methods used for energy evaluation on urban structures and sizing of building sites. These methods represent the ratio of incident solar irradiation on a building or land to unobstructed solar irradiation received at the same location during the chosen period of time, day or year, respectively [6]. The problem 
is the same for selection of solar power system installation places. However, the performance of the solar energy for buildings has been improved as the effects of the tree shading in some studies are taken into consideration $[7,8]$.

Environmental objects around the installation site decrease efficiency and productivity of the photovoltaic system. All shadings caused by buildings, mountains, and trees lead to early darkening of horizon. Therefore, heights of mountains, trees, and buildings far from or close to the installation area must be evaluated by means of a shading analyzer along with daily and yearly solar data in order to determine their effects on productivity and efficiency of the solar power system. To this aim, many existing devices with software, hardware, or software and hardware are used together [9]. On the other hand, there are many studies on new intelligent methods used for feasibility analysis of solar power installation and environmental impacts resulting from the established solar power plants $[10,11]$.

In this study, some of the existing shading analyzers are examined and introduced briefly. Afterwards, a different cost-efficient shading analyzer and site evaluator device together with developed software are presented and explained. Denizli-Yassihoyuk region, in Turkey, is selected as a case study. Simulation and experimental results are also given and discussed. It is demonstrated that the proposed system enables researchers to determine shades surrounding solar power system and analyze their effects on the system efficiency.

\section{Shading Analysis Tools}

The shadowing effects of obstructions such as trees, houses, or mountains cause reduction in duration of the sun exposure for PV system. For this reason, local surroundings of the installation site must be evaluated through taking annual sun path diagrams into consideration in order to determine the efficiency of the system to be established. First of all, duration of shading resulting from obstructions must be defined. There are some devices developed to analyze shading effects up to now. In the systems using only hardware equipment, yearly shading graph is drawn over the yearly sunlight chart for the local area. Afterwards, all of the obtained data can be evaluated by special software, which is called a solar path finder [12]. Data on surroundings recorded beforehand by the users are needed in the systems with only software unit. In addition, yearly sunlight database is maintained by another ancillary program like Google Earth. Some examples of these systems are Amethyst [13], Sombrero [14], and SUNDI [15].

The shading analyzers using software and hardware units use real-time data. The data on the surroundings can be transferred to any computer via an interface. Sun Eye is an example of these devices [16].

In addition to three types of shading analyzers, the proposed device in this study is also easy to apply and costefficient. The shading effects of environmental obstructions on efficiency of the solar power system are not taken into account in many of the mentioned devices due to their structures. However, environmental obstructions located remotely to the installation site influence the sunshine duration, exposure to sun, and solar energy input. Most of these devices contain lots of complicated hardware parts and the software developed by the users cannot be used independently.

\section{Solar Path Finder and Site Evaluator}

Solar path finder devices have been used in solar industry for shading analysis for decades. Instant panoramic reflection of the site provides accurate solar and shading data throughout the year. Solar path finder devices are nonelectronic instruments and measure the shading of any site, allowing the user to see what could shade the system throughout the year by means of their reflective principles. Additionally, the actual position of the sun is irrelevant with respect to the analysis time. In fact, units can be easily used in the absence of direct sunlight. An important component of the solar path finder is a transparent and highly polished convex plastic dome on top of a solid base. The user looks down onto the dome to see panoramic reflection of the site. All of the obstructions restraining the sunlight at the location can be clearly seen in the reflection. The sun path diagram representing the sun's route across the sky for each month of the year and hour of the day is drawn on a paper which is placed underneath the dome. Slots in the side of the dome allow the user to trace the outline of the reflected obstructions onto the diagram with a colored pencil and to determine the obstructions which will shade the selected installation site and its exact times, as shown in Figure 1. Although the analyzer system is simple, its compass shows the magnetic north. Therefore, it is necessary to know the deviation angle of installation site and to include all calculations. Besides, the obstructions located remotely to the power system and changing the shading factor cannot be sensed precisely.

Some of the site evaluator devices take a panoramic picture covered by mountains on the horizon line and distant obstructions. These pictures are transferred to a computer in order to evaluate them together with annual sun path diagrams. Asset and PV Watts [17] are well-known examples of the software parts used to upload the pictures on the computer and to combine the sun path maps as shown in Figure 2.

\section{Horizon Catchers and Real-Time Sunlight Observers}

Horizon catchers contain a digital camera, a horizon mirror, analyzing software, tripod, compass, and rectification unit as shown in Figure 3 as a model. These devices take a panoramic picture of the horizon and the obstructions surrounding the installation site [18].

The pictures including the horizon and obstructions are used to determine the solar energy input, sunshine duration, and exposure to sun reduced by obstructions such as trees, houses, or mountains by means of the special software of these devices. The analysis must be performed on the site. The catcher types are easy and fast tools to take outdoor pictures of the horizon although the pictures require a special computer 

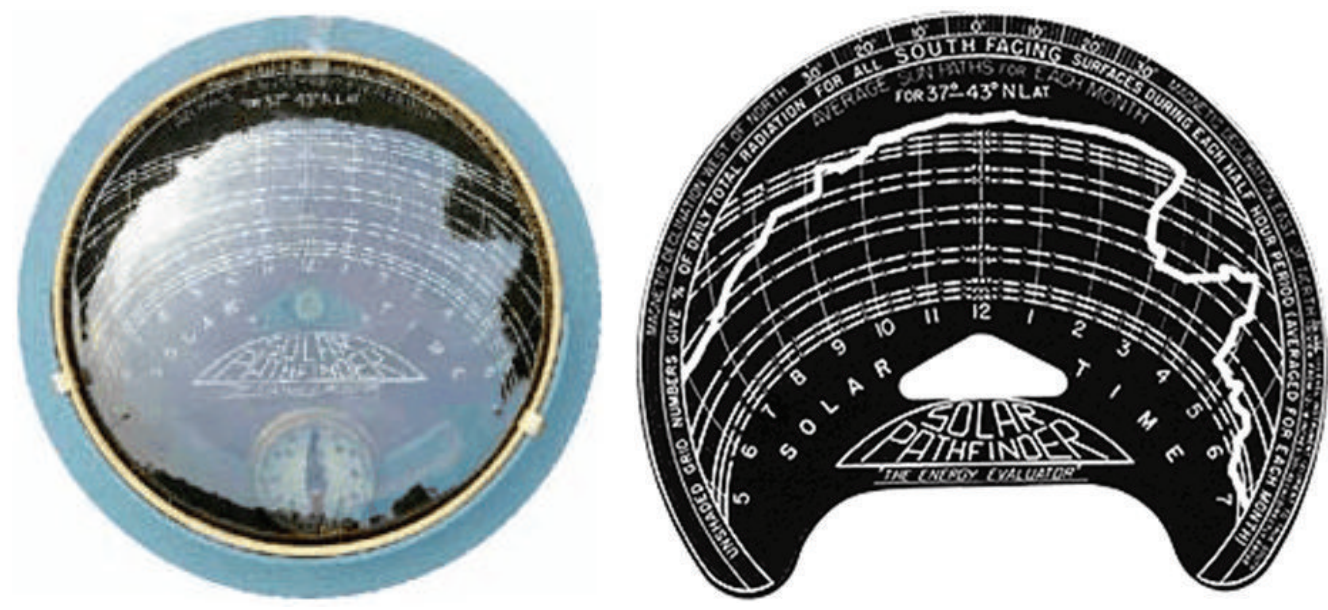

Figure 1: A typical solar path finder device (photo courtesy of Solar Pathfinder).

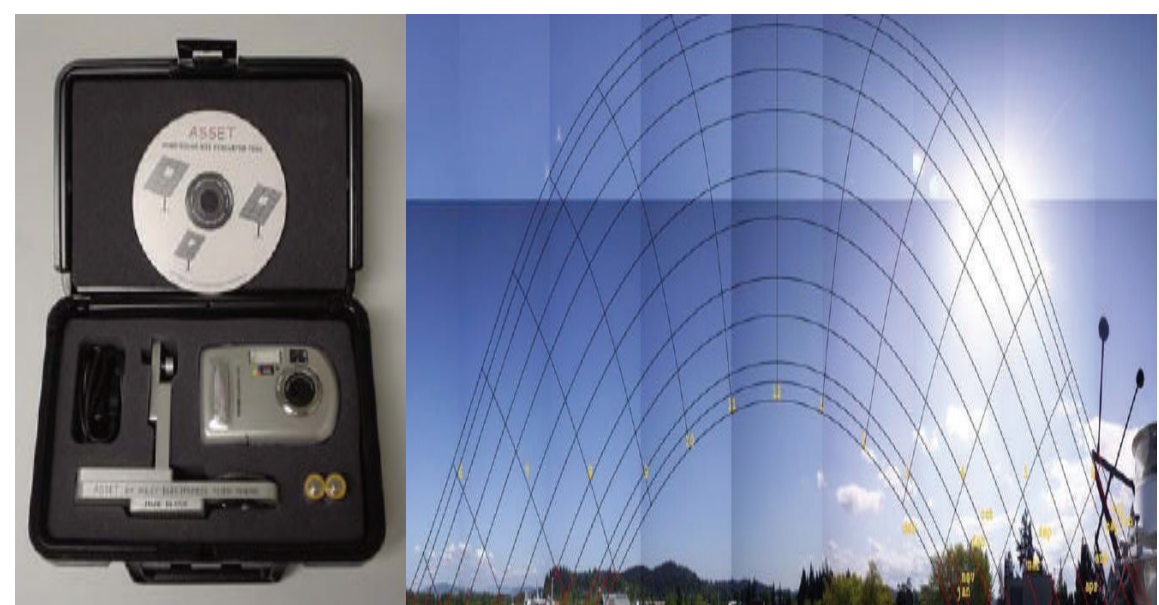

Figure 2: The Asset tools and series of the $9 \times 1$ pictures taken by them (photo courtesy of Solar Pathfinder).

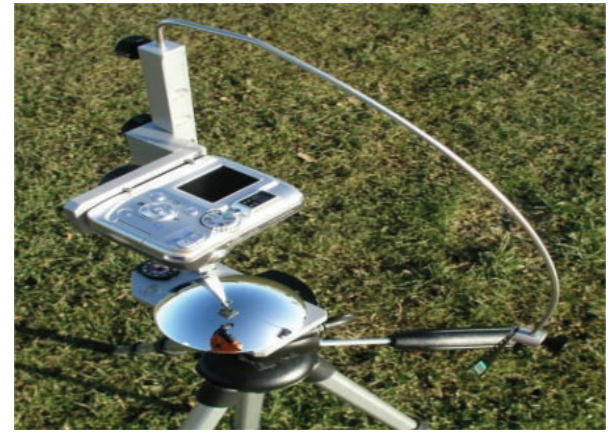

FIGURE 3: A typical horizon catcher device (photo courtesy of HORIcather).

program using local solar path diagrams. Furthermore, the difference angle between magnetic and geographic north is corrected manually.

As shown in Figure 4, some of the horizon catchers contain only electronic equipment such as a mounted digital camera using fish-eye lens, portable hardware, an electronic

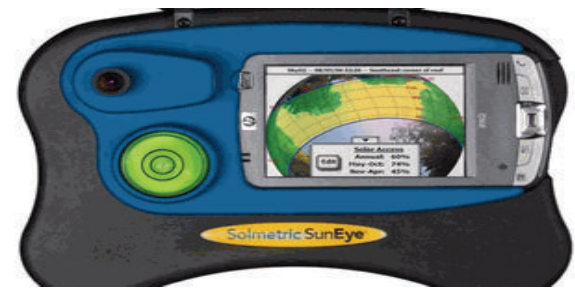

Figure 4: An electronic horizon and obstacle catcher (photo courtesy of Sun Eye).

compass, an optional GPS module, and embedded software. These device types are more useful but are more expensive than the others. However, these devices enable fast and accurate shading analysis of the site and export reports suitable for printing or send results to users as an e-mail.

\section{Trackers and Special Software for Mobile Phones}

This type of software can perform site evaluation and identifies the suitability of the site for solar power installation 


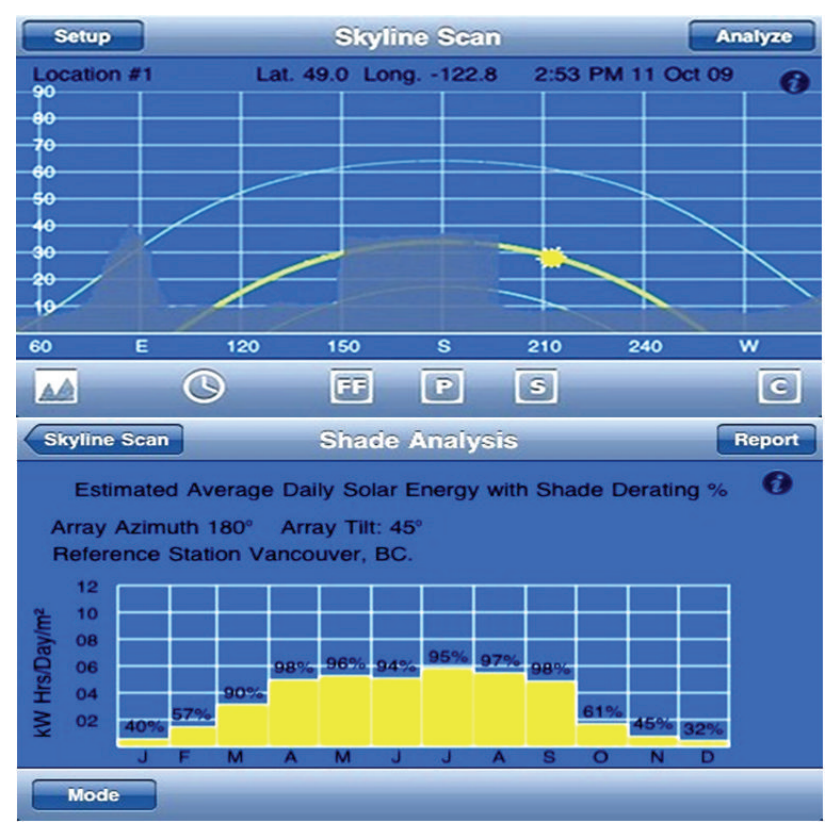

FIGURE 5: A typical skyline scanning and shading analysis by mobile phone.

as well as allowing users to calculate the solar energy and hours of direct sunlight on the installation site. It is the special application integrating solar energy calculation and shading analysis. In addition, it can be simplified in order to trace the outline of local skyline, to produce a trace of obstruction elevation angle for each degree of heading, and to display this along with trajectory of the sun. However, the users must set the tilt and position of the solar array. Afterwards, the program calculates the position and elevation of the sun throughout a full year. When the location is located under direct sunlight and the sun is shaded by local obstructions, it can be determined through using calculated data within the database of the weather station. Additionally, it can be used as typical weather data to identify daily solar potential of the installation site.

In addition, this application includes a large database of PV panels and inverters which makes the system configuration easier for the users [19]. However, this system which can evaluate real estate is only for iPhone and Android operating system as shown in Figure 5.

\section{A New Design for Shading Analysis}

In the conventional system, a protractor and a plumb bob are adequate enough to measure the altitude angle of an object as shown in Figure 6. However, an encoder generating 4095 pulses is used instead of protractor to measure the altitude angle in the proposed system. Thus, the altitude angle of any obstruction can be calculated precisely with a degree of 0.08789 in each vertical measuring step. The horizontal angle in panoramic view of the obstruction can be measured with the same sensitivity too. For this purpose, the second encoder is used to record the horizontal position of the obstruction by

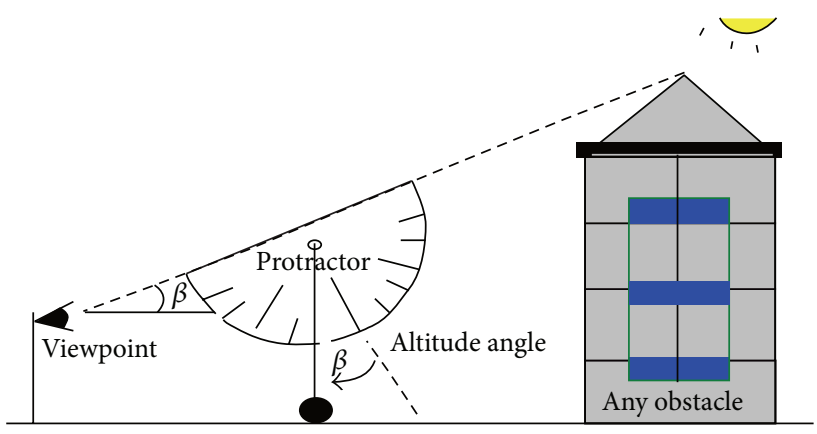

FIGURE 6: Using a protractor and plumb to measure altitude angle for any obstacles such as mountains, buildings, and trees.

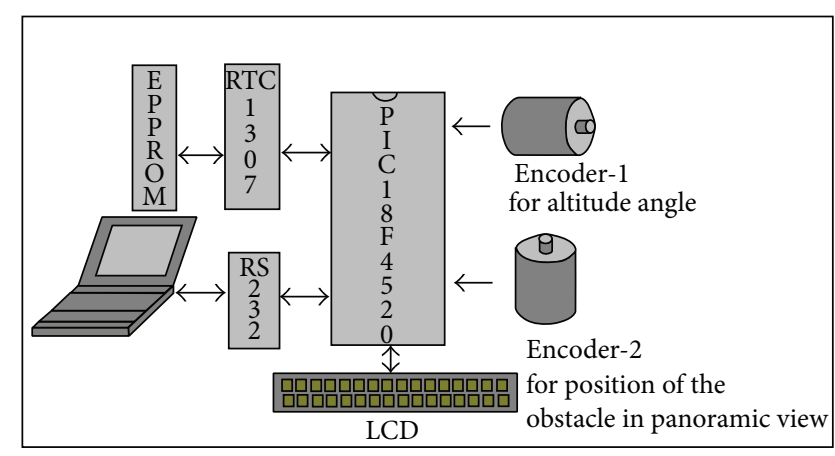

FIGURE 7: The principal model of the proposed device for shading analysis.

360 degrees' panoramic view with one degree more at each step.

\section{Fundamentals of the Proposed System}

The encoders used to sense the vertical and horizontal position of the obstruction generate data pulses in every measuring step and send these data to the microprocessor including real-time clock integrated into circuit on the control board of the analyzer tool. Measured positions and angles combined with clock data are transferred to a computer thanks to the serial port supported by the microprocessor. The developed system models are shown in Figures 7 and 8. The system includes a small telescope to view and track the top of the obstruction and two encoders to achieve altitude angle and horizontal position of the obstruction in 360degree panorama and microprocessor embedded software developed particularly for this study to evaluate received data and to perform data transfer processing.

By means of the small telescope on the device, obstacles located remotely to the installation site can be observed easily and determined accurately. Turning around self-axis by tracking top points of all obstructions surrounding the installation site, all of the measured data are recorded instantly by the microprocessor.

In addition to this, the system includes a display unit in order for the user to see altitude angle or horizontal position angle of the obstruction without using any PC software. The 


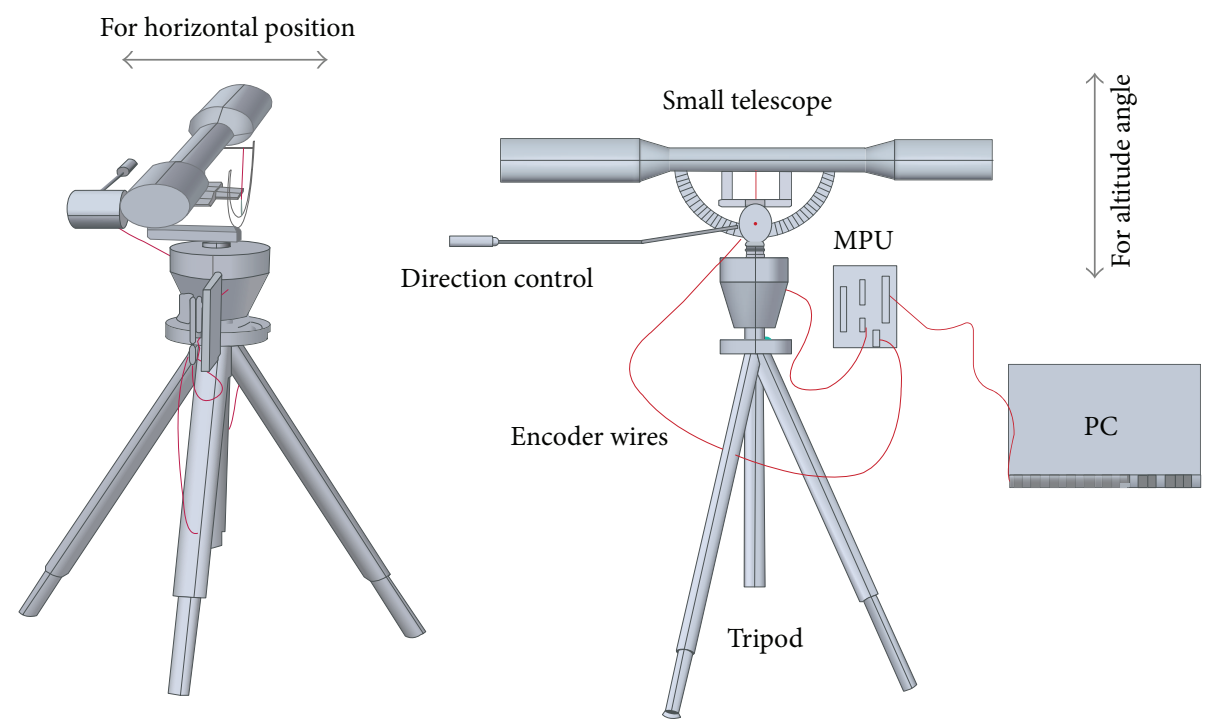

FIGURE 8: Introduction model of the proposed shading analyzer and site evaluator.

implemented device for site evaluation and shading analysis are shown in Figure 9, and implementation circuit is shown in Figure 10.

In order to determine geographic north, the angle of the sun on horizontal axis in accordance with the data received by a real-time clock (RTC) is calculated by the system after the user enters latitude and longitude values of the local site to the system. Afterwards, the lens of the telescope is directed to the sun and focuses on it and the position of the sun is recorded at that moment.

When the angle seen on the display is equal to zero, the geographic north is determined with respect to the sun. The other method to find geographic north in this system is to determine the magnetic north and to adjust the local rectification angle for the site which has been placed in the processor beforehand. In addition, the implemented system can be used with any evaluation software (like SUNDI, etc.) easily without encountering any problems.

\section{Determining the Sun Paths for the Software}

The position of the sun in space at a particular time instant can be calculated with respect to the longitude and latitude around the world. Moreover, the position of the sun can be precisely calculated with respect to the trajectory of the world via some equations. In other words, if the time, longitude, and latitude information is available, the position of the sun with respect to the application area can be calculated accurately.

Firstly, the declination angle of the world turning on the trajectory around the sun is between +23.45 and -23.45 degrees. This angle changes at an interval of very small degrees from year to year. However, the declination angle, $\delta$, can be calculated by different method as given in $[9,20]$

$$
\begin{aligned}
\delta & =\delta_{0} \cdot \sin \left(360 \frac{(284+n)}{365}\right) \\
& =23.45 \cdot \sin \left(\frac{360}{365}(n-81)\right),
\end{aligned}
$$

where $\delta_{0}$ is equal to $23.45^{\circ}$ and $n$ is day number of the year. For instance, it is equal to 45 on 14 February. The position of the sun at any time of the day is expressed by its altitude angle $\beta$ and azimuth angle $\varphi_{s}$ :

$$
\begin{gathered}
\sin \beta=\cos L \cdot \cos \delta \cdot \cos H+\sin L \cdot \sin \delta, \\
\sin \phi_{S}=\frac{\cos \delta \cdot \sin H}{\cos \beta} .
\end{gathered}
$$

To obtain sunrise and sunset times, $\beta$ is assumed as zero and $H$ (taking into account hour angle) as given by (3), which is the difference of the angles between local meridian (line of the longitude for the application area) and the sun's meridian (at any moment) with positive values obtained in the morning:

$$
H= \pm\left(\frac{15^{\circ}}{\text { hour }}\right) \quad \text { (hour before or after SN) . }
$$

Solar time, ST, is calculated as follows:

$$
\mathrm{ST}=\mathrm{CT}+\frac{4 \mathrm{~min}}{\operatorname{deg}}(\mathrm{LTM}-\mathrm{LL})^{\circ}+\operatorname{EOT}(\min ) .
$$

The solar noon, $\mathrm{SN}$ is obtained by relationship between civil and solar time as given equation (5),

$$
\mathrm{CT}=\mathrm{ST}-\frac{4 \mathrm{~min}}{\operatorname{deg}}(\mathrm{LTM}-\mathrm{LL})^{\circ}-\operatorname{EOT}(\min ),
$$

where CT is called local time or solar noon or clock/civil time in different literatures. The solar time ST is equal to 12:00, which is local standard noon. The equation of the time, EOT, is obtained by

$$
\mathrm{EOT}=9.87 \sin 2 B-7.3 \cos B-1.5 \sin B,
$$

where $B$ is given as follows:

$$
B=\left[\frac{360}{364}(n-81)\right] .
$$




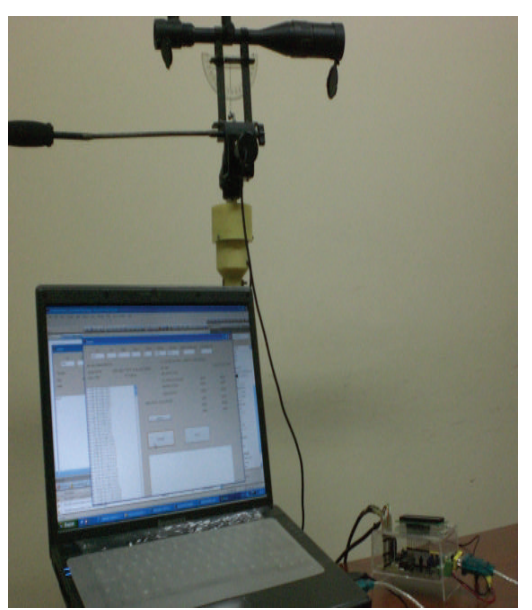

(a)

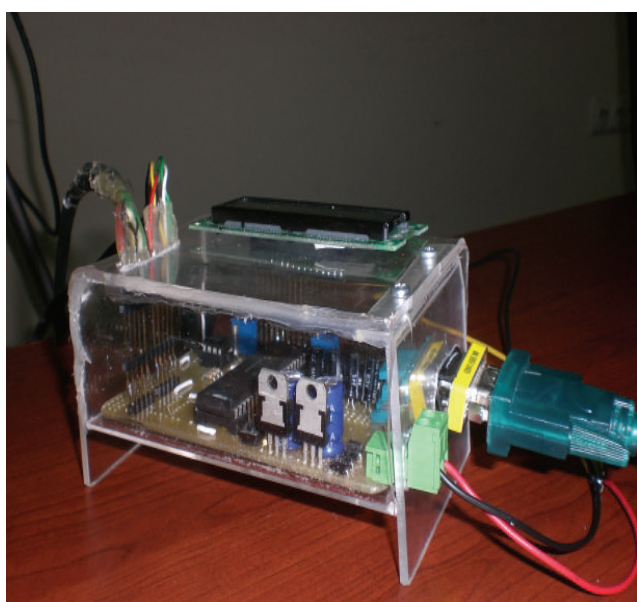

(b)

FIgURE 9: The implemented device for shading analysis (a). Interface card (b).

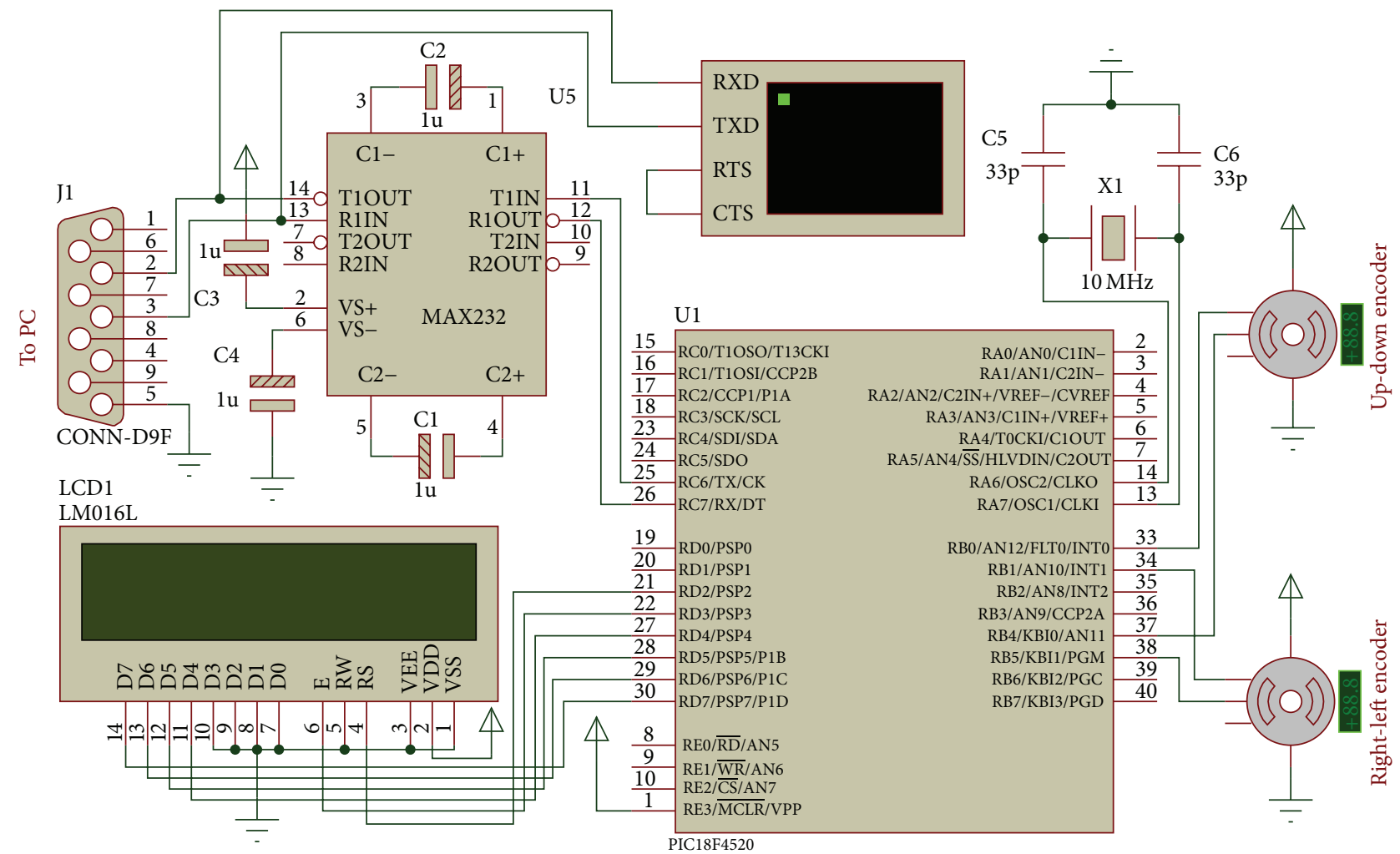

FIGURE 10: Implementation of open model for the proposed system.

An hour to solar noon, when CT is 12:00 on any day of the year, $H=+15^{\circ}$ for the time is eleven $\mathrm{AM}$ and $H=-30^{\circ}$ for the time is two PM. $\beta$ is equal to zero and hour angle is $H=$ HSR when the sun rises and sets:

$$
\begin{gathered}
\sin \beta=\cos L \cos \delta \cos H+\sin L \sin \delta=0, \\
\cos H=-\frac{\sin L \sin \delta}{\cos L \cos \delta}=-\tan L \tan \delta,
\end{gathered}
$$

where $H=\mathrm{HSR}$ is obtained by (9) as follows:

$$
\text { HSR }=\cos ^{-1}(-\tan L \cdot \tan \delta) .
$$

The sunrise and sunset time angles are obtained by HSR calculation along with sign of it. The sign of the HSR is positive at sunrise negative at the sunset. Taking into consideration the fact that $\mathrm{CT}$ equals 12:00, the sunrise 


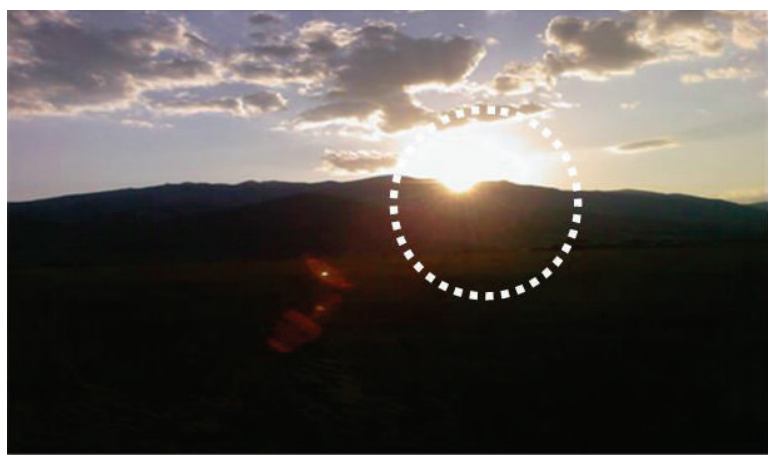

(a)

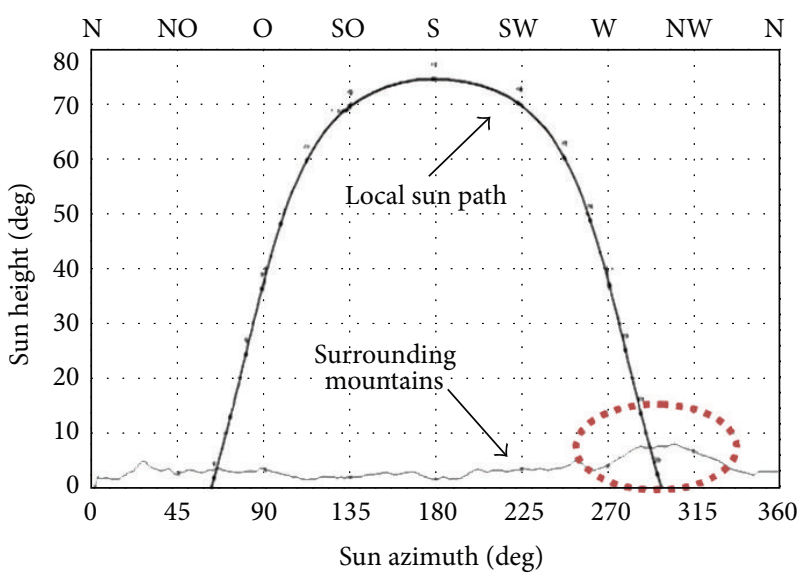

(b)

FIGURE 11: An analysis for application day. (a) A picture from the selected area; (b) recorded data of surrounding mountains combined with local sun path from developed software.

and the sunset times are calculated geometrically by (11) as follows:

$$
\text { GHSR }=12.00-\frac{\text { HSR }}{15} .
$$

GHSR value gives us the time when the center of the sun is on the horizon called the geometrical sunrise time. However, the angle between center of the sun and top of the sun with respect to local time zone is taken into account when the center of the sun is on the horizon. Furthermore, the reflection of the sunlight on atmosphere from the sun must be considered in the calculation of the GHSR in order to obtain true astronomical time values both at the sunrise and at sunset times.

Due to atmospheric refraction, which bends the sun's rays, the sun appears about $2.4 \mathrm{~min}$ sooner than geometrical sunrise and then 2.4 min later than sunset (this must be added or subtracted from GHSR).

The interval between the view of the sun on the horizon and the top of the sun is taken as changeable time with respect to the day of the year and location, which is called adjustment factor. The adjustment factor is extracted from GHSR value since the top of the sun appears firstly when the sun rises and is added to GHSR value since the top of the sun appears last when the sun sets. The adjustment factor is calculated by

$$
Q=\frac{3.467}{\cos L \cos \delta \sin (\mathrm{HSR})} \text {. }
$$

\section{Application and Results}

The model presented in this study is developed by means of local facilities and is open to further improvements when compared to three other models, namely, solar path finder and site evaluator, horizon catchers and real-time sunlight observers, and trackers and special software for mobile phones. In addition, this is the first study in Turkey which is a rich region in terms of solar energy. Although software used in this model yields quite positive results, it is still open to further improvements.

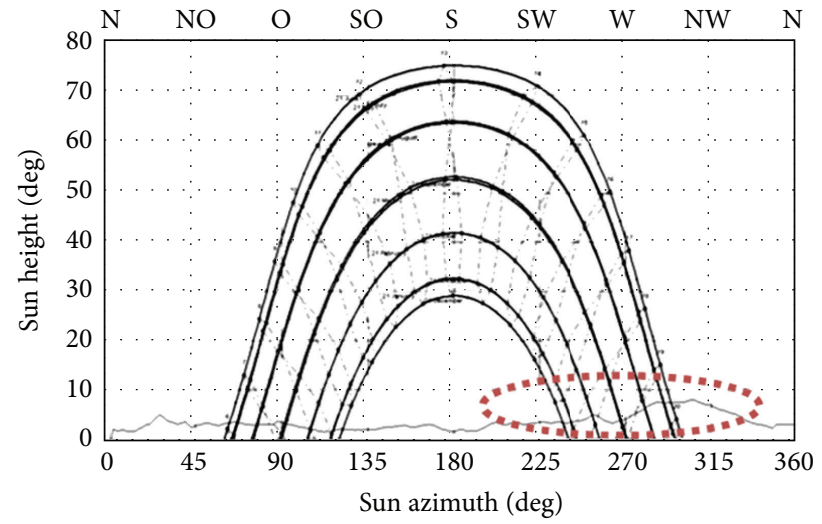

FIGURE 12: Annual sun paths and surrounding mountains in the selected area.

Yassihoyuk County of Denizli in Turkey is selected as a pilot area for solar power installation site and for testing the proposed system. The picture shown in Figure 11 was taken at 19:30 on May 26, 2013. The sun sets over the horizon of 292 degrees at the mentioned time.

After recording the environmental obstruction through the proposed system, the sun path diagram for the installation site is drawn by the software developed for the proposed device and is combined with the prerecorded data in order to prepare the shading graph. The obtained results are compatible with the picture, as shown in Figures 11 and 12.

When the measures are evaluated by the whole sun paths during the year, efficiency of the system to be established can be calculated and it can be decided whether the installation site is suitable for solar power system or not.

\section{Evaluation Software and Results}

Solar energy applications increase in Turkey day by day. However, the determination of the location for a solar power system still requires advanced knowledge and experience. 


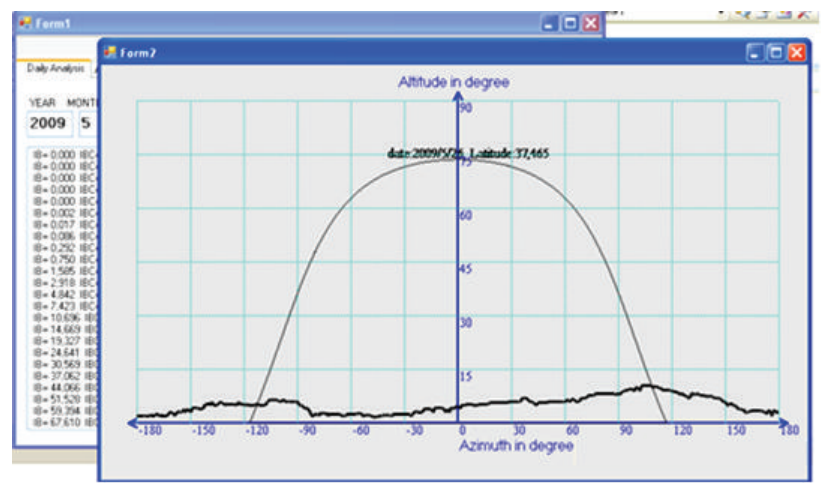

FIGURE 13: The screenshot of the developed software for application day.

This can be performed via various devices. The device offered in this study will pioneer upcoming investments in Turkey. Shading is one of most important factors influencing efficiency of PV systems. It is of vital importance to calculate shading. It is difficult to calculate shading factor via conventional methods because the sun's position varies depending on the season. The sun's movements are modeled and shading factors are measured thanks to the developed software. When the annual radiation rate of the location where PV system is to be established is known, it is possible to calculate the potential energy to be produced by that system.

As the data recorded for any installation site by means of the implemented system can be evaluated by any Office program, evaluation and monitoring software based on Microsoft Visual C\# platform is developed in order to estimate the effect of the shadows on the power system efficiency. The harvesting values of the sunlight with or without shadow can be estimated and displayed on a computer thanks to this interface program. Besides, the user can access local latitudelongitude data, the south angle and tilt angle of the PV panels. Some previews of the interface program developed for this study are shown in Figures 13 and 14.

\section{Conclusion}

In order to reduce consumption of the fossil fuels used in producing electricity and to decrease their emission of greenhouse gasses, existing renewable energy sources should be taken into consideration urgently and analyzed more. As far as location is concerned in PV systems, shading factor is as influential as coordinates. Shading objects surrounding the PV system affect the radiation value as much as its location. It is difficult to calculate shading factor via conventional methods because the sun's position varies depending on the season. The sun's movements are modeled and shading factors are measured thanks to the developed software. When the annual radiation rate of the location where PV system is to be established is known, it is possible to calculate the potential energy to be produced by that system. In this respect, a shading factor of 2 percent leads to enormous energy losses in a large PV system. Therefore, it is necessary to calculate radiation value in the location where PV systems

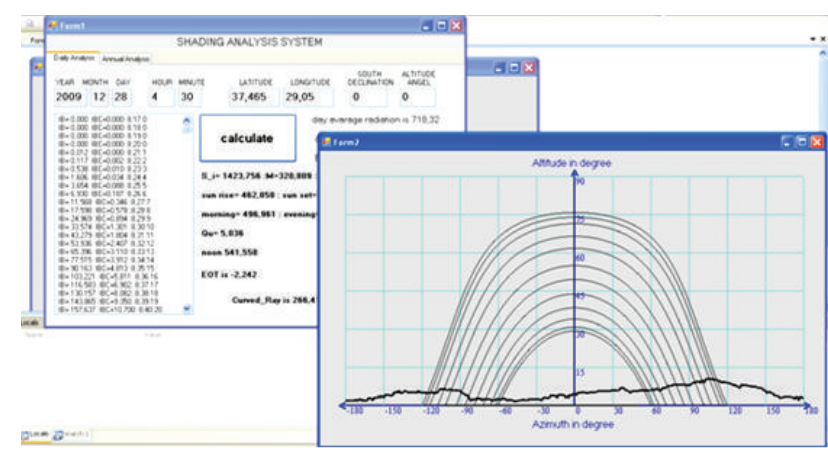

FIGURE 14: The screenshot of the developed software for the whole year.

are to be installed. This study is one of the most important studies which deal with the calculation of shading factor in Turkey. Software used in this model yields quite positive results and, moreover, it is still open to further improvements. Moreover, people who attempt to establish a new solar power system and take advantage of solar power should comprehend the importance of maximization of solar power production efficiency. Analysis of shading effects on solar power plant is one of the maximizing techniques to increase efficiency. This study presents a review on the existing shading analyzers and introduces a new shading analyzer and site evaluator based on a small telescope using two encoders as well as describing interface program extensively. The features of the newly designed device and newly developed software are compared with the others [21-23].

The proposed model operates independently of any interface program and it has the ability to identify distant obstructions on the site where the solar power system will be installed. The obstruction revealed by horizontal and vertical encoders can also be transferred to any computer via the hyperterminal by means of the developed microprocessorbased interface card. The system can be used very easily and rapidly. In addition, the obstructions on the site are identified more accurately degree by degree or at a sensitivity of less than one degree with respect to the users specific needs. Additionally, the proposed model in this study is a fairly costefficient model.

\section{Nomenclature}

B: $\quad$ Solar altitude angle

CT: $\quad$ Civil or clock time

EOT: Equation of time

$\phi_{S}: \quad$ Solar azimuth angle (+ before $\mathrm{SN},-$ after $\mathrm{SN}$ )

HSR: $\quad$ Sunrise and sunset hour angle

NOAA: National Oceanic and Atmospheric Administration

n: $\quad$ Day number of the year

GHSR: Geometrical sunrise and sunset hour angle

SST: $\quad$ Sunset time

Q: $\quad$ Adjustment factor

L: $\quad$ Latitude

LTM: Local time meridian 
LL: Local longitude

SN: Solar noon

ST: Solar time

SRT: Sunrise time

$H$ : Hour angle.

\section{Conflict of Interests}

The authors declare that there is no conflict of interests regarding the publication of this paper.

\section{Acknowledgments}

The authors would like to thank the editors and anonymous reviewers for their suggestions to improve the paper.

\section{References}

[1] N. S. Lewis, "Toward cost-effective solar energy use," Science, vol. 315, no. 5813, pp. 798-801, 2007.

[2] S. Rustemli and F. Dincer, "Modeling of photovoltaic panel and examining effects of temperature in Matlab/Simulink," Electronics and Electrical Engineering-Kaunas: Technologija, vol. 3, no. 109, pp. 35-40, 2011.

[3] J. Isenberg and W. Warta, "Realistic evaluation of power losses in solar cells by using thermographic methods," Journal of Applied Physics, vol. 95, no. 9, pp. 5200-5209, 2004.

[4] G. Hu, S. Duan, T. Cai, and C. Chen, "Sizing analysis of PV system with VRB storage," Electronics \& Electrical Engineering, vol. 118, no. 2, pp. 43-48, 2012.

[5] S.-H. Yoo and E.-T. Lee, "Efficiency characteristic of building integrated photovoltaics as a shading device," Building and Environment, vol. 37, no. 6, pp. 615-623, 2002.

[6] Ž. Kristl and A. Krainer, "Energy evaluation of urban structure and dimensioning of building site using iso-shadow method," Solar energy, vol. 70, no. 1, pp. 23-34, 2001.

[7] J. R. Simpson, "Improved estimates of tree-shade effects on residential energy use," Energy and Buildings, vol. 34, no. 10, pp. 1067-1076, 2002.

[8] G. Papadakis, P. Tsamis, and S. Kyritsis, "An experimental investigation of the effect of shading with plants for solar control of buildings," Energy and Buildings, vol. 33, no. 8, pp. 831-836, 2001.

[9] G. M. Master, Renewable and Efficient Electric Power Systems, John Willey \& Sons, New Jersey, NJ, USA, 2nd edition, 2004.

[10] E. Kabalci, "Development of a feasibility prediction tool for solar power plant installation analyses," Applied Energy, vol. 88, no. 11, pp. 4078-4086, 2011.

[11] D. Turney and V. Fthenakis, "Environmental impacts from the installation and operation of large-scale solar power plants," Renewable and Sustainable Energy Reviews, vol. 15, no. 6, pp. 3261-3270, 2011.

[12] R. Perez, B. Schultze, and J. Pryor, "Things that work: home power tests the solar pathfinder," Home Power Magazine, vol. 16, pp. 44-45, 1990.

[13] "Amethyst Shadowfx V3," 2012, http://www.shadowfx.com/.

[14] Sombrero, A PC-tool to calculate shadows on arbitrarily oriented surfaces, 2012, http://nesal.uni-siegen.de.

[15] SUNDI Simulation Program, 2012, http://www.web.co.bw/sib/ sundi_description.pdf.
[16] SunEye 210 Shade Tool, 2012, http://www.solmetric.com/.

[17] Asset, 2012, http://www.we-llc.com/.

[18] Horicatcher, 2012, http://www.meteotest.ch/en/footernavi/solar_ energy/horicatcher/.

[19] Solmetric Ipv App., 2012, http://www.solmetric.com/solmetricipv.html.

[20] A. Luque and S. Hegedus, Handbook of Photovoltaic Science and Engineering, John Wiley \& Sons, New York, NY, USA, 2003.

[21] S. Kesler, S. Kivrak, F. Dincer et al., "The analysis of PV power potential and system installation in Manavgat, Turkeya case study in winter season," Renewable \& Sustainable Energy Reviews, vol. 31, pp. 671-680, 2014.

[22] S. Rustemli, F. Dincer, E. Unal, M. Karaaslan, and C. Sabah, “The analysis on sun tracking and cooling systems for photovoltaic panels," Renewable and Sustainable Energy Reviews, vol. 22, pp. 598-603, 2013.

[23] S. Yilmaz, H. R. Ozcalik, O. Dogmus, F. Dincer, O. Akgol, and M. Karaaslan, "Design of two axes sun tracking controller with analytically solar radiation calculations," Renewable and Sustainable Energy Reviews, vol. 43, pp. 997-1005, 2015. 

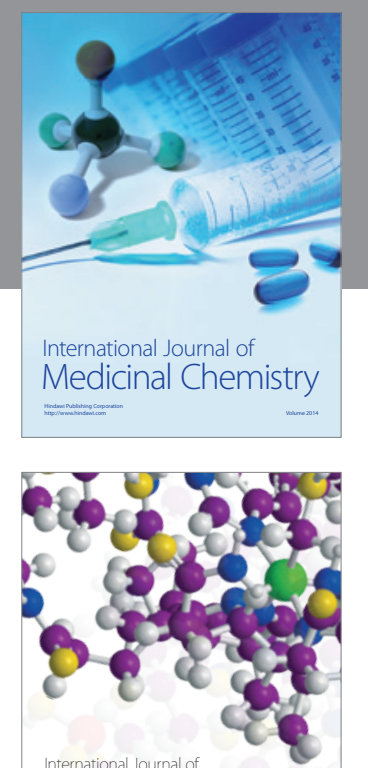

\section{Carbohydrate} Chemistry

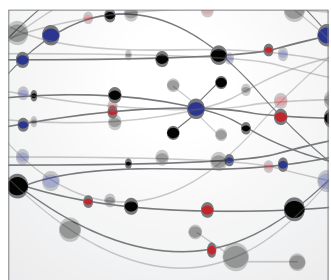

The Scientific World Journal
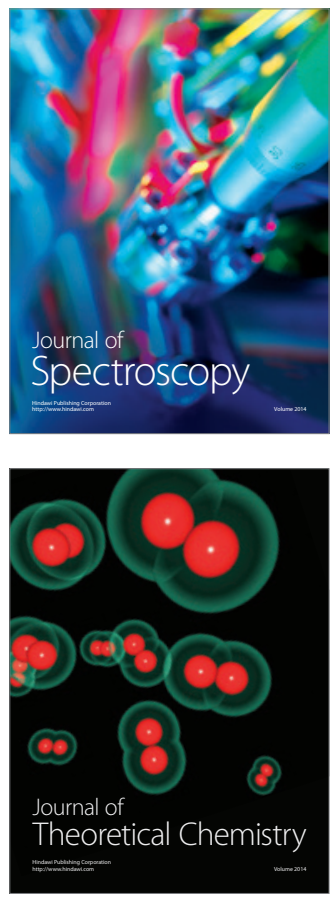
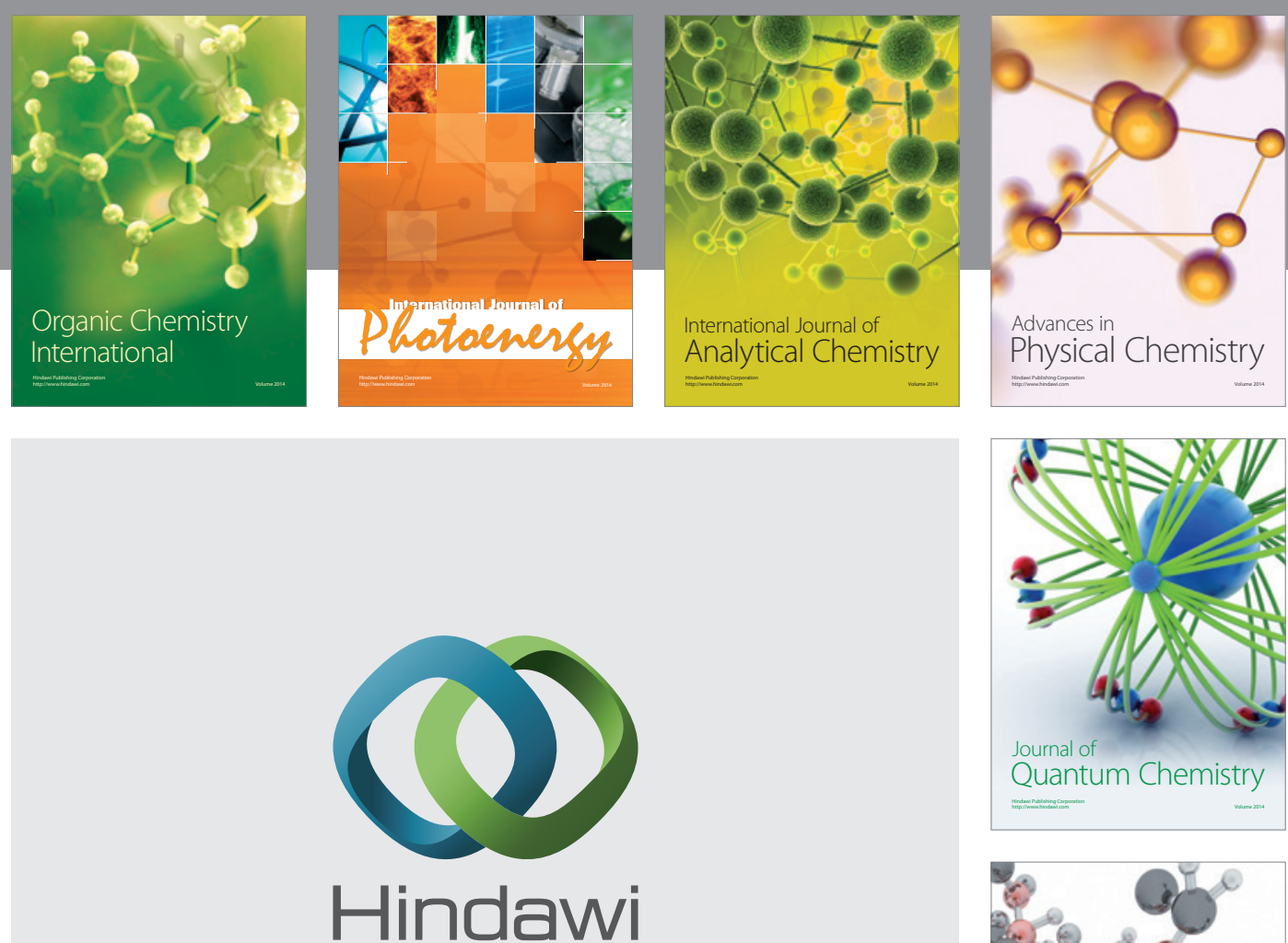

Submit your manuscripts at

http://www.hindawi.com

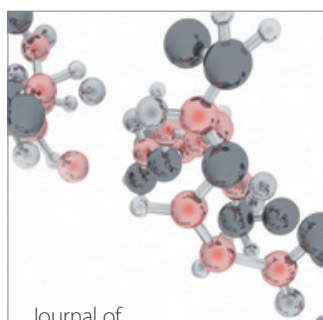

Analytical Methods

in Chemistry

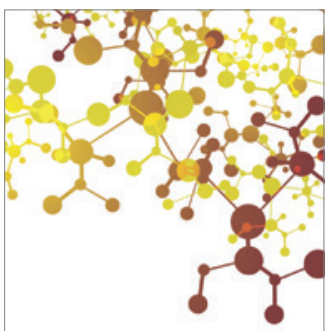

Journal of

Applied Chemistry

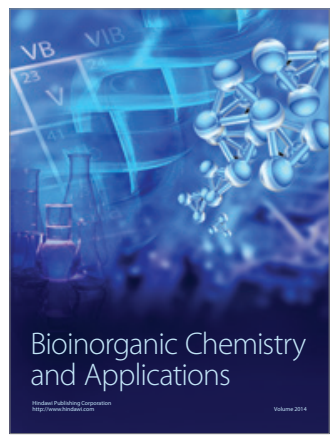

Inorganic Chemistry
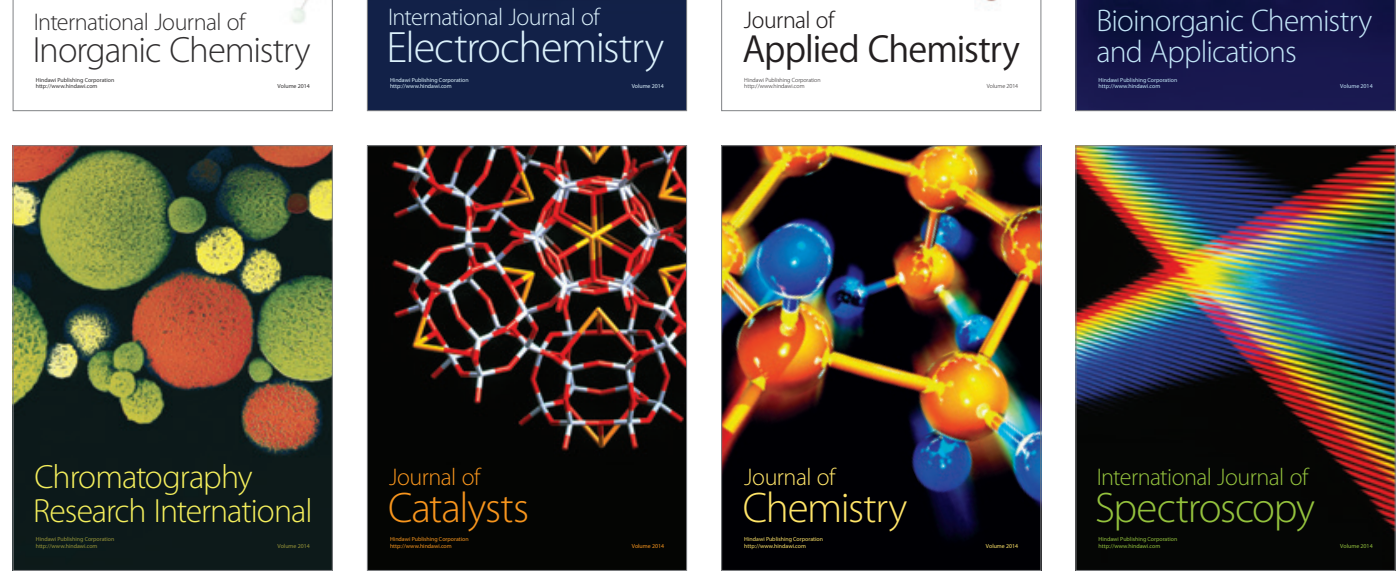